\author{
B. Świeczko-Żurek, W. Serbiński, A. Szumlański ${ }^{1}$ \\ Gdańsk University of Technology, Faculty of Mechanical Engineering, \\ ${ }^{1}$ Hospital in Inowrocław
}

\title{
ANALYSIS OF THE FAILURE OF FIXATOR USED IN BONE SURGERY
}

\begin{abstract}
The Gamma nail (fixator) made of stainless steel, which has broken in the body, was subjected to examination. The fixator was removed from the patient femur. The fracture occurred in the area of the nail hole after two months' stay in the organism. The research included chemical composition, hardness of the Gamma nail material and the observation and analysis of the type of fracture. The research showed the fatigue failure of the Gamma nail.
\end{abstract}

Key words: Gamma nail, stainless steel, fatigue failure

\section{INTRODUCTION}

The fundamental role of an implant is performing some definite functions in organism corrections and repairs of organ disfunctions. It may happen, however, that due to some factors an implant will not be able to perform its role in a proper way. The loss of functional abilities may occur as a result of a mechanical damage of an implant, postoperation complications and infections. Resulting in the rejection of implant by an organism.

Surgical treatment of fractions involves indirect invasion into trauma environment, which has an influence on natural course of bone healing. According to literature concerning surgical treatment of shaft of femur fractions with adults there is possibility of local complications, despite applying different osteosynthesis methods and having good treatment results. To avoid complications the least invasive method of surgical treatment should be chosen. The healing process of fracture ought to be similar to natural one [1]. The choice of the most infective method depends on different factors. The kind of fracture, the age and general condition of a patient, the condition of soft tissue surrounding the damaged bone, the set of implants. The surgical experience of the team is of paramount importance. Intramedullary osteosynthesis is the method which gives big possibility of effective operation, with relatively rare occurrences of local and general complications.

Before being produced every implant is subjected to many laboratory examinations, which define the implants ability to transfer the loading for relatively long time. While performing the examination on the machines imitating the movements of an implant in 
an organism the item is acted on by forces several times bigger than the ones in natural environment. Unfavourable impact of forces may cause early consumption or total destruction of the implant [7]. Fatigue damage of intramedullary nails (fixators), which will be discussed in further parts of the article is a serious problem of different types of stabilization. The main kinds of damage observed in clinic practice are [2]: fractions in cross-section spots, fractions close to holes as well as plastic deformation along the nail. The construction of some nails makes it possible to perform the interfragmental compression for some kind of fractures in the course of the operation [3]. For the biomechanical properties of knitting the important things are: the thickness and the diameter, the shape of the section as well as the material of which the implant was made. The nails are usually bent, the radius of curvature of a normal nail A0 is $1,5 \mathrm{~m}$ and corresponds to anatomical features of the bone shaft structure [4].

The Gamma nails (Fig.1) make it possible to cure complicated comminuted fractures, fractures of neck of femur as well as intertrochanteric fractures and pathological ones. They must transform loadings caused by forces on head of femur, which bend position crew, entering the nail and introduced through the neck into the head of femur [2].

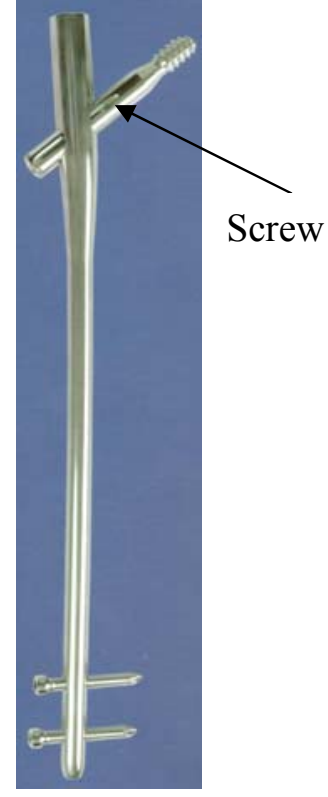

Fig. 1. The Gamma nail [2]

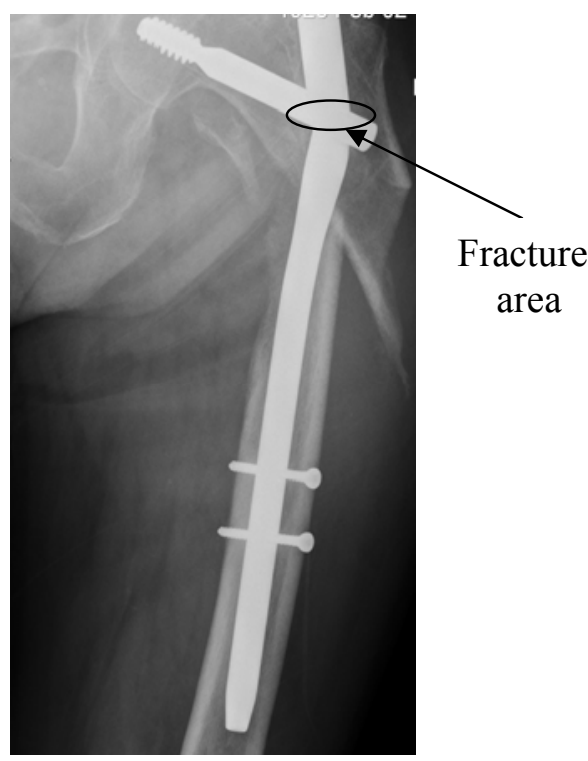

Fig. 2. The picture of Gamma nail in the leg after breaking

The intramedullary nails belong to short-term implants. The time of their duration in the body does not surpass two years. For that reason, that kind of implants is made of stainless steel, which has good mechanical properties and high corrosion resistance.

Intramedullary nailing is a relatively little invasive method, as it does not require the opening the natural environment. The bone knitting is quick. The location of a nail in medullar canal close to the mechanical axis of femur diminishes the risk of implant damage and enables an early start of limb loading [5].

However, if a patient does not obey the doctor's instructions concerning recovery period, the trauma banding or breaking of fixator may occur. This paper presents a broken Gamma nail, the analysis of its destruction and application instructions. 


\section{EXPERIMENTAL AND DISCUSSION}

The object of research was the broken Gamma nail, whose location and fastening in the leg was shown in X-Ray examination (Fig. 2).

The analysis of Gamma nail fracture started with macroscopic observation of the outer surface as well as the whole surface in the fracture area. No signs of mechanical or corrosion destruction were observed on the outer surfaces. While some dishes in the whole surface were spotted in the fracture area. It was plastic deformation of the nail in the contact place with the screw. The chemical composition of the Gamma nail material was examined by means of the Scanning Electron Microscope Philips XL 30, equipped with devices to analyse the chemical composition (EDS), and the results were presented as a spectrum and the print in Fig. 3.

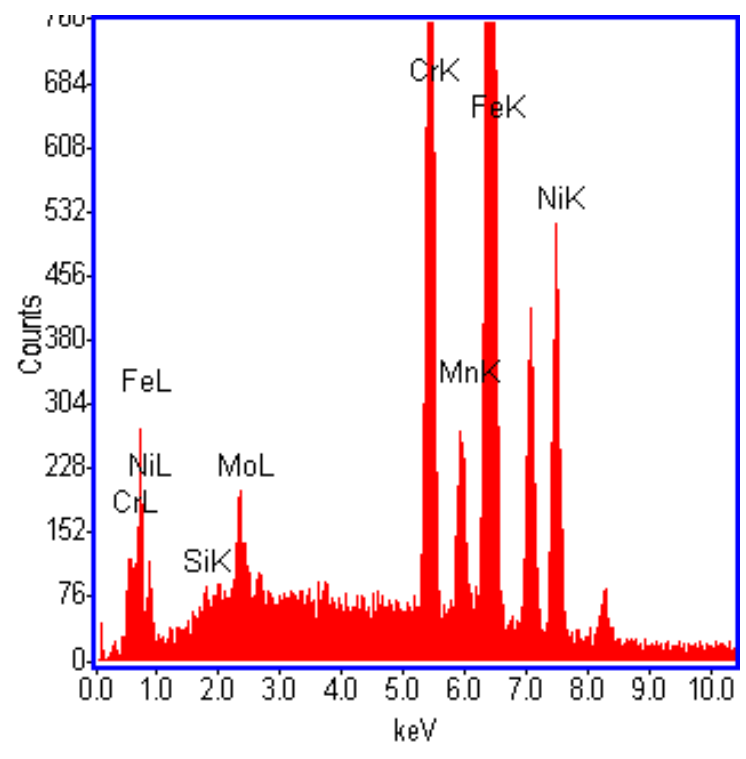

\begin{tabular}{crr} 
Element & Wt o & \multicolumn{1}{c}{ At 8} \\
\hline SiK & 0.58 & 1.16 \\
CrK & 17.57 & 19.09 \\
MnK & 1.93 & 1.99 \\
FeK & 59.97 & 60.68 \\
NiK & 14.29 & 13.75 \\
MoK & 5.66 & 3.34 \\
Total & 100.00 & 100.00
\end{tabular}

\begin{tabular}{lrcrr} 
Element & Net Inte. & Bkgd Inte. & Inte. Error & \multicolumn{1}{c}{ P/B } \\
\hline SiK & 2.34 & 4.71 & 14.66 & 0.50 \\
CrK & 151.88 & 6.61 & 0.85 & 22.98 \\
MnK & 13.25 & 5.77 & 3.76 & 2.30 \\
FeK & 358.53 & 5.32 & 0.54 & 67.39 \\
NiK & 58.28 & 4.06 & 1.40 & 14.35 \\
MoK & 2.07 & 1.38 & 10.62 & 1.50
\end{tabular}

Fig. 3. The spectrum and the chemical composition of the Gamma nail material

The chemical composition analysis proves that, the material of the examined nail is stainless Cr-Ni-Mo steel. According to the Schäffler's diagram [6], the austenitic structure of the examined material was found. Its hardness was defined by means of Vickers method of measurement by loading of 294,2 N. The average hardness of the Gamma nail is presented in Table 1 . 
Table.1. The hardness HV30 of the intramedullary nail

\begin{tabular}{|c|c|}
\hline The Gamma nail element & The average hardness HV30 \\
\hline Nail & 321 \\
\hline Screw & 320 \\
\hline
\end{tabular}

The examination of fixator breaking was restricted exactly to the area of breaking, which is shown in Fig. 2.

The observations of the fatigue fracture were performed using the SEM with different magnifications (from $23 \mathrm{x}$ to $1000 \mathrm{x}$ ). The chosen views of the fatigue fractures are presented in Fig. 4.

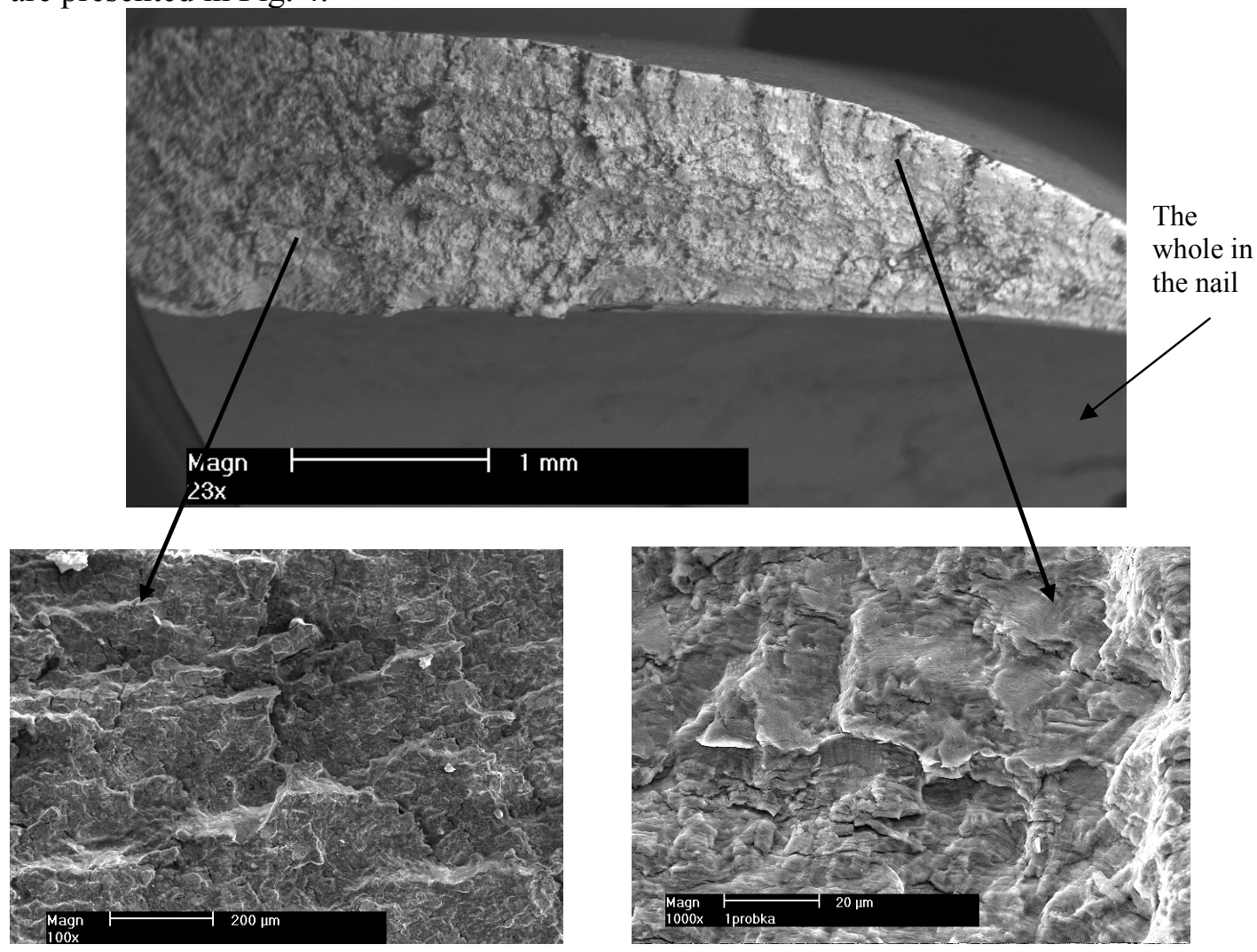

Fig. 4. View of the fatigue fracture of the Gamma nail

It was found that both the nail and the screw cooperating with it are equally hard (320 HV30), which proves the proper combination of the materials in the construction of the examined nail.

The observations of the fixator (Gamma nail) fracture show that failure has fatigue nature with characteristic focus of the stresses concentration. The analysis of biomechanical loading of the nail (Fig. 2) and the fracture (Fig. 4) indicates that concentration of variable stresses most probably appeared of the whole edge in the Gamma nail. It can't be excluded that the course of fatigue fracture might have been started by micro fracture caused by the impact of loading (for example a fall) and its further advancement in the course of further use of the examined Gamma nail. 


\section{CONCLUSIONS}

1. The tested material of the Gamma nail was made of Cr-Ni-Mo stainless steel, commonly used in trauma surgery.

2. The failure of the Gamma nail showed the fatigue nature.

3. The fatigue fracture was initiated of the whole edge at the contact with the screw.

4. During the rehabilitation period patients should absolutely adhere to doctor's instructions, dealing with moving and loading a broken bone that was stabilized by the Gamma nail.

\section{REFERENCES}

1. Wójcik K., Nowak R., Wroński S., Wojciechowski P., Służałek M.: Estimation of post- operation treatment of the shaft of femur fraction. Movement Organ Surgery, Polish Orthopedics, 64 (1): 31-38, 1999.

2. Van Doorn R.: Patients treated with the long Gamma nail. Castellum 2001.

3. Mittelmeier H., Mittelmeier W., Grabowski M.T.W.: The new compressioninterlocking nail used for knitting of long bones. Movement Organ Surgery, Polish Orthopedics, 57, Supl. 3: 45-47, 1992.

4. Seiler J.G., Swiontkowski M.F., A prospective evaluation of the AO/ASIF universal femoral nail in the treatment of traumatic and reconstructive problems of the femur. J. Trauma. 31 (1): 121-126, 1991.

5. Wójcik K.: The biomechanical aspects of intramedullary nailing in the shaft of treatment. Annales academiae medicae silesiensis., The II Symphosium Materials of Biomechanics in Implantology, Supl.29, 156-163, 1999.

6. Dobrzański L.A., Brytan Z., Grande M.A., Rosso M., Pallavicini E.J.: Properties of vacuum sintered duplex stainless steels. J. of Mat. Process. Techn., v. 162-163, 2005, 286-292.

7. Marciniak J., Chrzanowski W., Krauze A.: Intramedullary nail in osteosynthesis. Monograph, WPŚl., Gliwice 2006. 